Cahiers de la recherche sur les droits fondamentaux

12 | 2014

Droit et psychiatrie

\title{
La protection des malades mentaux par le droit civil
}

Gilles Raoul-Cormeil

\section{OpenEdition}

Édition électronique

URL : http://journals.openedition.org/crdf/1910

DOI : $10.4000 /$ crdf. 1910

ISSN : 2264-1246

Éditeur

Presses universitaires de Caen

Édition imprimée

Date de publication : 1 décembre 2014

Pagination : 59-72

ISBN : 978-2-84133-507-7

ISSN : 1634-8842

Référence électronique

Gilles Raoul-Cormeil, «La protection des malades mentaux par le droit civil », Cahiers de la recherche sur les droits fondamentaux [En ligne], 12 | 2014, mis en ligne le 01 octobre 2015, consulté le 11 février 2020. URL : http://journals.openedition.org/crdf/1910; DOI : 10.4000/crdf.1910 


\section{La protection des malades mentaux par le droit civil}

\section{Gilles RAOUL-CORMEIL}

Maître de conférences en droit privé à l'université de Caen Basse-Normandie

Centre de recherche en droit privé (CRDP, EA 967)

\section{Introduction}

A. Le malade mental, le mal-aimé du droit civil

B. La dignité du malade mental restaurée par l'idée de protection

C. La dignité du malade mental également restaurée par l'idée d'indifférenciation

D. L'ambiguïté de la protection du malade mental

II. La protection des biens du malade mental

A. Tournée vers l'avenir, la protection prospective des malades mentaux

1. La variété des techniques de protection des biens

2. L'équilibre entre le pouvoir de protection, le contrôle du protecteur et l'autonomie du protégé

B. Tournée vers le passé, la protection rétroactive des malades mentaux
1. Insania omnia corrumpit
2. La preuve du trouble mental
3. Le délai pour agir en nullité

III. La protection de la personne du malade mental

A. La protection au cours de l'ouverture de la mesure

1. L'institutionnalisation d'un médecin habilité

2. Le statut du médecin habilité

3. Les missions du médecin habilité

4. Le problème du refus du malade mental de se laisser examiner par le médecin

B. La protection au cours de la mise en œuvre de la mesure

1. La considération de la loi pour l'intimité du majeur protégé

2. Les droits simplement personnels

3. Les droits strictement personnels

IV. Conclusion 


\section{Introduction}

\section{A. Le malade mental, le mal-aimé du droit civil}

"Prince de l'exégèse ${ }^{1}$, "champion et chantre ${ }^{2}$ de la possession d'état pour établir la filiation de l'enfant né hors mariage, Jean Charles Florent Demolombe (1804-1887) fut aussi le promoteur du mandat de protection future ${ }^{3}$ et un ardent défenseur de la protection individuelle de l'aliéné ${ }^{4}$. Au XIX ${ }^{\mathrm{e}}$ siècle, sous l'empire du Code Napoléon, alors que l'interdiction judiciaire privait l'aliéné de sa capacité juridique, il soutint que l'interdit pouvait seul exercer la plupart des droits subjectifs qui étaient attachés à sa personnalité: se marier, tester, reconnaître un enfant naturel ${ }^{5}$. Victor-Napoléon Marcadé (1810-1854), à l'inverse, refusait toute exception à l'incapacité générale et admettait sans scrupule que l'interdiction puisse entraîner une incapacité générale de jouissance pour les actes éminemment personnels ${ }^{6}$. Le Code Napoléon n'envisageait que la représentation de l'aliéné dans les actes patrimoniaux. Pour le reste, le silence maintenu à l'égard des actes personnels suscitait donc la discussion sur l'étendue de l'incapacité générale. Une doctrine majoritaire s'inclinait par prudence devant le consentement manifesté par l'aliéné dans un intervalle de lucidité ${ }^{7}$, preuve la plus éclatante de la capacité naturelle de la personne humaine ${ }^{8}$, en professant que le fait relevait sans doute de l'hypothèse d'école. La référence discrète au droit naturel permettait de contenir une loi liberticide. Cette discussion révèle néanmoins la piètre considération dans laquelle le droit civil tenait les malades mentaux. «Cachez ces aliénés qu'on ne savait voir!» Le Code Napoléon contribuait à leur exclusion de la société. Il faudra attendre l'année 1968 pour que le Code civil ouvre ses bras protecteurs au malade mental.

\section{B. La dignité du malade mental restaurée par l'idée de protection}

Fruit de l'un des neuf avant-projets de loi rédigés par le doyen Carbonnier (1908-2003) pour réformer, un à un, les titres du livre premier du Code civil, la loi no $68-5 \mathrm{du}$ 3 janvier 1968 a modifié le paradigme de l'aliéné. En la forme d'abord, la loi nouvelle a abandonné la terminologie stigmatisante du Code napoléon. L'interdit judiciaire et le conseil judiciaire ont ainsi fait place à la trilogie aujourd'hui célèbre: tutelle, curatelle et sauvegarde de justice. Surtout, «l'imbécillité, la démence ou la fureur» ${ }^{9}$ ont été remplacées par une explication médicale et sociale: l'altération des facultés mentales causée par la «maladie, une infirmité ou un affaiblissement dû à l'âge $»^{10}$. Sous ce changement formel, l'idée perce au fond que les aliénés sont des sujets de droit à part entière. La sagesse du législateur dissimule à peine son humanité et sa clairvoyance : nul n'est assuré d'échapper à la dégénérescence de ses aptitudes intellectuelles et mentales.

Dans la lignée de Demolombe, Carbonnier a imprimé à la loi civile le but de protéger l'aliéné et non pas de le sanctionner. La loi de 1968 a permis aux malades mentaux

1. P. Malaurie, Anthologie de la pensée juridique, Paris, Cujas, 1996, p. 187 sq., spéc. p. 188. Adde, notamment, J. Musset, «Un célèbre jurisconsulte caennais du XIX ${ }^{\mathrm{e}}$ siècle, Demolombe», Revue trimestrielle de droit civil, 1995, p. 85.

2. G. Cornu, Droit civil: la famille, $9^{\mathrm{e}}$ éd., Paris, Montchrestien (Domat droit privé), 2006, nº 250, spéc. p. 350.

3. Voir J. Hauser, «Les mesures judiciaires, solutions subsidiaires au mandat de protection future?», in Nouveau droit des majeurs protégés: difficultés pratiques, G. Raoul-Cormeil (dir.), Paris, Dalloz (Thèmes et commentaires: actes), 2012, p. 13-24.

4. L'étymologie nous enseigne que le terme «aliéné» signifie l'étranger. Le droit civil en a gardé un sens précis. «Aliéner», c'est transmettre à autrui la propriété d'un bien. L'aliéné désigne également la personne qui a perdu sa raison. Par sa folie, elle est en quelque sorte étrangère à elle-même.

5. C. Demolombe, Cours de Code Napoléon, $2^{\mathrm{e}}$ éd., vol. 8. Traité de la minorité, de la tutelle et de l'émancipation, t. 2, Paris, A. Durand - Hachette, $1861, \mathrm{n}^{\circ} 643$, p. 434 sq.: "C'est qu'en effet l'interdiction totale et absolue, quand même! ne serait plus une mesure de protection, mais constituerait elle-même, dans son exagération une atteinte pleine de dureté et d'inhumanité aux droits les plus précieux du citoyen. Qu’y aurait-il, en effet, tout à la fois de plus inconséquent et de plus tyrannique qu'une loi qui déclarerait absolument incapable, en droit, de reconnaître un enfant naturel, de se marier, de tester, un individu qui serait, en fait, très-capable de consentir tous ces actes! qui l'en déclarerait incapable, non point par l'effet d'une déchéance pénale quelconque, mais dans un but de garantie et de protection!». Malgré le style décalé de la plaidoirie, perce ici, en avant-garde, toute la philosophie de la loi du 3 janvier 1968, à laquelle a été fidèle la loi du 5 mars 2007.

6. Voir ainsi à propos de l'article $146 \mathrm{du}$ Code civil, V.-N. Marcadé, Explication théorique et pratique du Code civil, $7^{\mathrm{e}}$ éd., t. 1, Paris, Delamotte et fils, $1873, n^{\circ} 520$, p. 408: "Après l'interdiction, l'individu est déclaré incapable d'avoir une volonté, et le mariage qu'il contracterait, même pendant un intervalle lucide, serait radicalement nul». Adde, sur l'ancien article 502 du Code civil, ibid., t. 2, Paris, Delamotte et fils, 1873 , n 320 , spéc. p. 321: «Le seul acte frappé de nullité proprement dite par suite de l'interdiction, c'est le mariage de l'interdit». Adde, dans le même sens, C.-B.-M. Toullier, Le droit civil français, suivant l'ordre du Code Napoléon, t. 1, Rennes, J.-M. Vatar, 1811, nº 448, p. 398.

7. Voir notamment M. Planiol, avec la collaboration de G. Ripert, Traité élémentaire de droit civil, 12 éd., t. 1, Paris, LGDJ, 1937, nº 709 , p. 267 ; R. T. Troplong, Le droit civil expliqué suivant l'ordre des articles du Code, t. 1. Du contrat de mariage, Paris, C. Hingray, 1850, nº 289 sq., p. $341-348$; T. Huc, Commentaire théorique et pratique du Code civil, t. 2, Paris, F. Pichon, 1892, nº 17 in fine, p. 28, et t. 3, Paris, F. Pichon, 1892, n 519 , p. 505; A. Vigié, Cours élémentaire de droit civil français, $2^{\mathrm{e}}$ éd., t. 1, Paris, A. Rousseau, 1893, nº 906, p. 510; A. Colin, H. Capitant, Cours élémentaire de droit civil français, $8^{\mathrm{e}}$ éd., Paris, Dalloz, 1934, nº 580 in fine, spéc. p. 609.

8. En ce sens, voir J.-M. Plazy, La personne de l'incapable, La Baule, La Mouette (Doctorat et notariat; 11), 2001 (préface de J. Hauser), nº 354 , p. 240.

9. Code civil, art. 493 (rédac. loi du 30 ventôse an XII (21 mars 1804)) : «Les faits d'imbécillité, de démence, ou de fureur, seront articulés par écrit. Ceux qui poursuivront l'interdiction, présenteront les témoins et les pièces». Adde, Code civil, art. 504 (rédac. loi du 30 ventôse an XII (21 mars 1804)): "Après la mort d'un individu, les actes par lui faits ne pourront être attaqués pour cause de démence, qu'autant que son interdiction aurait été prononcée ou provoquée avant son décès; à moins que la preuve de la démence ne résulte de l'acte lui-même».

10. Code civil, art. 490, al. 1 (rédac. loi du no 68-5 du 3 janvier 1968): «Lorsque les facultés mentales sont altérées par une maladie, une infirmité ou un affaiblissement dû à l'âge, il est pourvu aux intérêts de la personne par l'un des régimes de protection prévus aux chapitres suivants ». 
de se marier ${ }^{11}$, pourvu qu'ils y soient autorisés par leurs proches parents et puissent manifester un consentement lucide devant l'officier de l'état civil. La victoire de Demolombe sur Marcadé ne fut cependant pas totale. La thèse de Marcadé allait persister à propos du droit de tester $^{12}$ des malades mentaux placés sous le régime de tutelle que la loi de 1968 leur a très officiellement retiré jusqu'à la loi no 2006-728 du 23 juin 2006. Autorisés par un conseil de famille et représentés par un tuteur, les tutélaires pouvaient seulement transmettre leurs biens par donation, mais seulement à leurs descendants ou à leur conjoint. Cette donation où le donateur était représenté sans être consulté était un pacte de famille. Pour le reste, la loi de 1968 a maintenu le doute sur les actes éminemment personnels, laissant à chacun la pudeur de répondre si l'aliéné pouvait avoir des relations sexuelles et procréer ${ }^{13}$. La loi de 1968 avait néanmoins affiché la dimension protectrice du mécanisme de l'incapacité civile. La vocation du patrimoine de l'aliéné était de combler les besoins de son maître avant de retourner à sa famille par le sang.

Fille légitime de la loi du 3 janvier 1968 , la loi nº 2007308 du 5 mars 2007 a été fidèle à l'esprit et aux principes directeurs de la loi Carbonnier ${ }^{14}$. En témoigne l'article 415, alinéa 1 du Code civil, selon lequel «Les personnes reçoivent la protection de leur personne et de leurs biens que leur état ou leur situation rend nécessaire». Le dispositif de la protection des biens a été développé pour répondre à la variété des actes juridiques en usage. La protection de la personne sort complètement affirmée des travaux parlementaires. Le droit supranational des droits de l'homme aura aussi fortement influencé le législateur puisqu'il rattache la liberté personnelle du majeur protégé au maintien de ses droits fondamentaux ${ }^{15}$ et consacre à la question des actes de la vie personnelle du majeur protégé un faisceau de textes ${ }^{16}$.

\section{La dignité du malade mental également restaurée par l'idée d'indifférenciation}

Avec une sobriété exemplaire, la loi de 1968 avait déjà restauré la dignité de l'incapable majeur en engageant à son profit un profond mouvement d'indifférenciation de la personne. La grâce accordée aux aliénés naquit de la variété des causes médicales de leurs maux. Les malades mentaux ont disparu derrière l'étiquette neutre de majeur protégé. Le droit civil ne leur réserve plus une catégorie juridique spécifique ${ }^{17}$. Il leur ouvre un statut ou leur permet d'obtenir, tout simplement, l'annulation du contrat qu'ils auront conclu sous l'empire d'un trouble mental. Principe d'égalité oblige ${ }^{18}$, les malades mentaux répondent surtout - comme toute personne qui jouit de sa pleine lucidité et de sa pleine capacité juridique - de leur faute personnelle ${ }^{19}, \mathrm{du}$ fait des choses qu'ils ont sous leur garde et même du fait d'autrui, lorsque l'enfant mineur sur lequel ils exercent l'autorité parentale a causé un préjudice

11. Code civil, art. 506 (rédac. loi no 68-5 du 3 janvier 1968): «Le testament fait après ouverture de la tutelle sera nul de droit» (al. 1); «Il n’y a pas lieu à la réunion d'un conseil de famille si les père et mère donnent l'un et l'autre leur consentement au mariage» (al. 2); «Dans tous les cas, l'avis du médecin traitant doit être requis» (al. 3).

12. Code civil, art. 504 (rédac. loi nº 68-5 du 3 janvier 1968): «Même dans le cas des articles 497 et 499, le mariage d'un majeur en tutelle n'est permis qu'avec le consentement d'un conseil de famille spécialement convoqué pour en délibérer. Le conseil ne peut statuer qu'après audition des futurs conjoints» (al. 1); «Le testament antérieurement fait restera valable, à moins qu'il ne soit établi que, depuis l'ouverture de la tutelle, a disparu la cause qui a déterminé le testateur à disposer» (al. 2).

13. Sur le débat doctrinal qui a poursuivi sous l'empire de la loi du 3 janvier 1968, voir J. Hauser, «Réflexions sur la protection de la personne de l'incapable», in Mélanges Pierre Raynaud, Paris, Dalloz - Sirey, 1985, p. 227-236; T. Fossier, "Les libertés et le gouvernement de la personne incapable majeure", La semaine juridique, éd. G, 1985, I, 3195; J.-P. Gridel, L'acte éminemment personnel et la volonté propre du majeur en tutelle, in Rapports annuels de la Cour de cassation, 2000, p. 79-92; J. Massip, Tutelle des mineurs et protection juridique des majeurs, Paris, Defrénois-Lextenso, 2009, $\mathrm{n}^{\circ} 423$.

14. Parmi les commentaires de la loi du 5 mars 2007, voir T. Fossier, «La réforme de la protection des majeurs. Guide de lecture de la loi du 5 mars 2007", La semaine juridique, éd. G, 2007, I, 118, p. 13-23; P. Malaurie, «La réforme de la protection juridique des majeurs", Defrénois, 2007, art. 38569, p. 557-572; J. Hauser, «Des incapables aux personnes vulnérables», Droit de la famille, 2007, étude n 14, p. 5-7; «Le statut du majeur protégé après la loi du 5 mars 2007 ", actes du colloque tenu à l'université de Caen Basse-Normandie les 20 et 21 mars 2008 , sous la direction de A. Batteur, L. Mauger-Vielpeau, G. Raoul-Cormeil, la publication des dix-sept études étant répartie entre La semaine juridique, édition notariale, $\mathrm{n}^{\circ} 36,5$ septembre 2008, p. 17-65 et la Revue de droit sanitaire et social, $\mathrm{n}^{\circ} 5$, septembre-octobre 2008 , p. $807-850$, selon qu'elles portent sur un thème patrimonial ou social.

15. Code civil, art. 415, al. 2 (rédac. loi n ${ }^{\circ}$ 2007-308 du 5 mars 2007).

16. Code civil, art. 457-1 à 463 (rédac. loi nº 2007-308 du 5 mars 2007).

17. Sur l'essor de la catégorie de personne vulnérable, voir J. Hauser, «Les difficultés de la recodification: les personnes", in Le Code civil, 1804-2004: livre du bicentenaire, Paris, Dalloz - Litec, 2004, p. 201-218, spéc. p. 213. Adde, V. Mikalef-Toudic, A. Cermolacce, "Catégorie de personnes et capacité», in Différenciation et indifférenciation des personnes dans le Code civil. Catégorie de personnes et droit privé (1804-2004), P. Bloch, C. Duvert, N. Sauphanor-Brouillaud (dir.), Paris, Économica (Études juridiques; 23), p. 41-6o.

18. Par-delà sa valeur de droit constitutionnel, l'égalité est aussi un principe important du droit civil. Sur lequel, voir notamment D. Berthiau, Le principe d'égalité et le droit civil des contrats (thèse de doctorat, université Paris 2, juin 1997), Paris, LGDJ (Bibliothèque de droit privé; 320 ), 1999 (préface de J.-L. Sourioux); P. Jestaz, «L'égalité et l'avenir du droit de la famille», in L'avenir du droit: mélanges en hommage à François Terré, Paris, Dalloz - PUF, 1999, p. 417-430.

19. Code civil, art. 489-2 (rédac. loi nº 68-5 du 3 janvier 1968): "Celui qui a causé un dommage à autrui alors qu'il était sous l'empire d'un trouble mental n'en est pas moins obligé à réparation». Pour un commentaire, voir R. Savatier, "Le risque, pour l'homme, de perdre l'esprit et ses conséquences en droit civil», Dalloz, 1968, Chron., p. 109-116; G. Viney, «Réflexions sur l'article 489-2 du Code civil», Revue trimestrielle de droit civil, 1970, p. 251; P. Le Tourneau, «La responsabilité civile des personnes atteintes d’un trouble mental», La semaine juridique, éd. G, 1971, I, 2401. Ce texte a été déplacé à l'article 414-3 du Code civil par la loi nº 2007-308 du 5 mars 2007. 
à un tiers ${ }^{20}$. Cette forte manifestation de l'indifférenciation, entre les personnes saines d'esprit et les personnes insanes, a été possible parce que la dette de réparation d'un préjudice causé à autrui sanctionne le patrimoine et non plus la liberté de son titulaire et, surtout, parce que le risque de causer à autrui un préjudice dont il se serait rendu responsable peut être reporté sur une société d'assurance, obligée par un contrat synallagmatique et à titre onéreux, à indemniser la victime. Par-delà la réforme des institutions du droit des majeurs protégés, la loi de 1968 a permis un changement de regard sur le public des personnes dites vulnérables. Il suffit de songer au travail de fond engagé par les associations tutélaires fondées au lendemain de son entrée en vigueur.

\section{L'ambiguïté de la protection du malade mental}

Les malades mentaux continuent néanmoins d'incarner une "dissociation ${ }^{21}$. Leur personnalité demeure le théâtre d'un conflit intérieur entre un bourreau ${ }^{22}$ et une victime. Un conflit qui marque l'hésitation du législateur entre la promotion de sa liberté et la construction d'un régime de protection qui passe par l'enfermement. Le doyen Carbonnier nous avait déjà avertis du risque de dérive: «légiférer sur les fous, pour les fous, n'est-ce pas le pire? ${ }^{23}$. Le législateur a résolu ses doutes en installant des contre-pouvoirs. Ni le médecin, ni le juge ne doivent exercer leurs fonctions sans contrôle. Le savoir du premier est une limite au pouvoir du second. Comme le pouvoir accordé au premier en raison de son savoir médical ${ }^{24}$, dans la décision de retenir une personne contre son consentement en hôpital psychiatrique, doit être limité par le pouvoir juridictionnel du juge judiciaire ou administratif ${ }^{25}$. Portée par les meilleures intentions, l'idée de protection peut ainsi s'avérer excessive. Le risque de dérive n'est pas moins grand aujourd'hui qu'hier. Devant le nombre croissant de mesures de protection à l'égard d'une population vieillissante ${ }^{26}$, la loi du 5 mars 2007 réformant le droit des majeurs protégés a renforcé le triple principe: nécessité, subsidiarité, proportionnalité. La loi est très prudente dans l'ouverture d'une mesure de protection juridique. Il ne suffit donc pas de souffrir d'une altération de ses facultés mentales pour être placé sous un régime de curatelle ou de tutelle. Si le juge peut faire autrement, il devra privilégier les mesures alternatives que sont notamment la procuration, le mandat de protection future et la représentation judiciaire, si elles permettent de pourvoir aux intérêts de la personne vulnérable. En droit civil, la protection du malade mental ne passe donc pas toujours par l'instauration d'une incapacité de protection. Pour le démontrer, l'étude envisagera tour à tour les biens (II) et la personne du malade mental (III).

\section{La protection des biens du malade mental}

La protection des biens du malade mental est tournée vers l'avenir lorsqu'il bénéficie d'une mesure de protection qui le place officiellement sous un régime d'incapacité juridique. La protection sera tournée vers le passé lorsque le malade mental conservera sa pleine capacité juridique. Résolument inégale, la protection est donc prospective (A) ou rétroactive $(\mathrm{B})$.

\section{A. Tournée vers l'avenir, la protection prospective des malades mentaux}

\section{La variété des techniques de protection des biens}

La protection des biens d'une personne souffrant d'une altération de ses facultés mentales s'inscrit dans la durée. Il faut donc écarter la sauvegarde de justice car cette

20. CA Caen, 2 février 2006, $\mathrm{n}^{\circ} \mathrm{RG}$ 05/00663. Adde, G. Raoul-Cormeil, «L'incapable majeur civilement responsable du fait de son enfant mineur », Dalloz, 2006, Jur., p. 2016-2019.

21. Rappr. F. Terré, préface à M.-P. Champenois-Marmier, J. Sansot, Droit, folie, liberté: la protection de la personne des malades mentaux (loi du 30 juin 1838), Paris, PUF (Travaux et recherches de l'Université de droit, d'économie et de sciences sociales de Paris), p. 15-17, spéc. p. 16: «Le fou étant divisé en deux, la thérapeutique devrait logiquement se dérouler sous le contrôle de celui qui parlerait au nom de l'aliéné. La définition communément admise du trouble mental, envisagé comme une dissociation, n'appelle-t-elle pas dans la perspective d'un remède, l'existence de deux personnes?».

22. Dont pâtissent parfois les membres de leur famille. Sur l'impossibilité d'appliquer le droit des majeurs protégés à l'encontre du majeur protégé, voir G. Raoul-Cormeil, «La famille du majeur protégé: un environnement protecteur, destructeur ou victime», Cahiers de la recherche sur les droits fondamentaux, $\mathrm{n}^{\circ} 11,2013$, p. 63-75.

23. J. Carbonnier, Essais sur les lois, $2^{\mathrm{e}}$ éd., Paris, Répertoire du notariat Defrénois, 1995, p. 63-78, spéc. p. 71: «Mais légiférer sur les fous, pour les fous, n'est-ce pas le pire? Très vite l'impression doit vous venir qu'on ne peut être pour eux, sans au fond, d'une certaine manière, agir contre eux. Car toute protection des aliénés, en un sens, les aliène, par cela seul qu'elle les suppose étrangers à l'univers raisonnable. Si bien que, procédant des meilleures intentions, une législation protectrice ne fera qu'ajouter de son métal à cet appareil répressif par lequel les sociétés modernes, avec des hypocrisies variables, rejettent de leur sein ceux qui ont le malheur d'avoir des mécanismes mentaux dissidents".

24. Sur la corrélation entre savoir et pouvoir, voir A. Sériaux, «La notion de doctrine juridique», Droits, $\mathrm{n}^{\circ} 20,1994$, Doctrine et recherche en droit, p. 65-74. Adde, en matière médicale: A. Catherine, Pouvoir du médecin et droits du patient: l'évolution de la relation médicale, thèse de doctorat en droit public, université de Caen Basse-Normandie, 2011 (dactyl.), p. 2-19, à propos de la notion de «pouvoir médical», de ses significations et de son ampleur.

25. Outre les travaux publiés dans les actes du colloque «Droit et psychiatrie» du 15 novembre 2011, voir A. Bertrand-Mirkovic, «L'hospitalisation d'un majeur protégé sans son consentement", in Nouveau droit des majeurs protégés..., p. 173-182, où sont étudiés la genèse et les apports de la loi $\mathrm{n}^{\circ}$ 2011-803 du 5 juillet 2011.

26. J. Favard, «La protection des personnes âgées vulnérables», in Droit et vieillissement de la personne, J.-R Binet (dir.), Paris, LexisNexis-Litec (Colloques \& débats; 21), 2008, p. 7-10. 
mesure provisoire ne dure qu'une année, sauf si le juge des tutelles fixe une durée plus courte. Ce délai d'un an est renouvelable une fois pour la même durée et sous certaines conditions ${ }^{27}$. Alors que la loi de 2007 a défini la sauvegarde de justice comme une mesure autonome, appropriée au besoin de protection d'une personne dont l'esprit est obscurci de manière passagère, la pratique des juges des tutelles continue à l'utiliser à des fins d'instruction, le temps d'arbitrer entre une mesure de curatelle, simple ou renforcée, et une mesure de tutelle. Seules les mesures de curatelle et de tutelle peuvent être prises pour une durée de cinq $a n s^{28}$ et, lors de leur révision, peuvent être exceptionnellement renouvelées pour une durée plus longue aujourd'hui déterminée ${ }^{29}$. Le principe de nécessité oblige le juge des tutelles à adapter la mesure au besoin de protection de la personne. Posée à l'article 428, alinéa 2 du Code civil, la règle de la proportionnalité est une règle corollaire du principe de nécessité. En pratique, les juges des tutelles n'ont pas toujours le loisir, ni la connaissance précise de la situation du majeur à protéger pour tailler sur mesure un régime de protection, comme les y invite la loi. La sauvegarde de justice, avec ou sans mandataire spécial, la curatelle simple ou renforcée, et la tutelle constituent un éventail de mesures standards et graduées ${ }^{30}$.

Chacune de ces mesures est animée par une technique majeure et différente qui lui confère une nature propre. Ainsi la technique de la surveillance caractérise la sauvegarde de justice. La technique de l'assistance correspond à la curatelle et celle de la représentation est de l'essence de la tutelle. Intermédiaire, la curatelle renforcée est aussi un régime complexe. D’un côté, le curateur représente le curatélaire, comme dans la tutelle aux biens, pour percevoir ses revenus et payer ses dépenses courantes ${ }^{31}$, sans qu'il soit nécessaire de distinguer entre les actes d'administration et de disposition. Mais, d'un autre côté, pour les autres actes juridiques, tels que la vente d'une maison, la souscription d'une assurance sur la vie, la conclusion d'un emprunt immobilier ou d'un crédit à la consommation, l'engagement d'une action en justice pour faire annuler un acte patrimonial, le curateur ne peut plus représenter le curatélaire. Les règles de la curatelle simple retrouvent leur force obligatoire ${ }^{32}$. L'assistance chasse la représentation. L'intérêt du curatélaire sera exprimé par la double signature du curatélaire et du curateur.

\section{L'équilibre entre le pouvoir de protection, le contrôle du protecteur et l'autonomie du protégé}

Si l'on prend l'hypothèse d'une personne souffrant d'une grave altération de ses facultés mentales, le choix se fera entre la curatelle renforcée et la tutelle, ce qui n'est pas neutre quant à la protection des biens ${ }^{33}$. Dans les deux cas, la technique de la représentation permettra au tuteur ou au curateur de faire face aux dépenses courantes: loyer, paiement des charges du logement ou d'une pension alimentaire, constitution des dossiers de demandes de prestations sociales et familiales et perception des revenus. Les biens du majeur incapable seront ainsi protégés parce qu'il ne pourra pas les engager par sa seule signature. La protection devrait atteindre son but.

Mais à bien y réfléchir, la protection et la représentation paraissent incompatibles entre elles. Généralisée à tous les actes, la technique de la représentation aboutirait, dans sa mise en œuvre, à entretenir ou même à aggraver l'état d'aliénation du majeur dit protégé. Aussi, contre cet excès de représentation, il faut évoquer deux garde-fous.

D'abord, pour les actes patrimoniaux les plus graves, c'est-à-dire ceux que la loi qualifie d'acte de disposition ${ }^{34}$, le tuteur devra solliciter l'autorisation d'un juge des tutelles qui pourra, en auditionnant le majeur protégé, l'associer à la décision. Si la technique de la représentation judiciaire permet de se passer du majeur protégé pour la signature de l'acte, il est de bonne pratique de toujours l'associer à la décision. La parole du majeur protégé doit être provoquée et entendue, bien qu'elle ne lie ni le tuteur, ni le juge des tutelles. L'ouverture d'une mesure de protection juridique a pour effet de créer une distance entre la volonté du tutélaire et son intérêt. Alors que l'intérêt de toute personne pleinement capable est réputé atteint par l'expression de sa volonté, le majeur protégé pourrait agir, eu égard à l'altération de ses facultés mentales, contre son intérêt. Le risque est avéré en tutelle. Gardien de cet intérêt, le juge des tutelles délègue alors à un tuteur la préservation de l'intérêt du tutélaire lorsqu'il lui confie la mesure de protection. Mais les tuteurs s'astreignent à consulter les tutélaires pour définir leur intérêt. Telle est la règle déontologique observée par les mandataires judiciaires à la protection des majeurs (MJPM) qui bénéficient d'un monopole lorsque le juge des tutelles écarte

27. Code civil, art. 439, al. 1 (rédac. loi no 2007-308 du 5 mars 2007).

28. Code civil, art. 441 (rédac. loi $n^{\circ}$ 2007-308 du 5 mars 2007).

29. Code civil, art. 442, al. 2 (rédac. loi no $2007-308$ du 5 mars 2007). La loi de 2007 a fait cesser la pratique des mesures de protection juridique prises à durée indéterminée et parfois vécue comme une mort civile. Voir, en ce sens, M. Rebourg, «Durée et renouvellement des mesures de protection juridique: le pragmatisme des juges face à la loi», in Nouveau droit des majeurs protégés..., p. 37-48, spéc. p. 38.

30. Code civil, art. 440 (rédac. loi n ${ }^{\circ} 2007-308$ du 5 mars 2007).

31. Code civil, art. 472 (rédac. loi no $2007-308$ du 5 mars 2007).

32. Voir notamment N. Peterka, A. Caron-Déglise, F. Arbellot, Droit des tutelles: protection judiciaire et juridique des mineurs et des majeurs, Paris, Dalloz (Dalloz référence), 2012, $\mathrm{n}^{\circ}$ 75-21.

33. Ni quant à la protection de la personne, voir infra (III).

34. Code civil, art. 505. Adde, décret no 2008-1484 du 22 décembre 2008, art. 2: «Constituent des actes de disposition les actes qui engagent le patrimoine de la personne protégée, pour le présent ou l'avenir, par une modification importante de son contenu, une dépréciation significative de sa valeur en capital ou une altération durable des prérogatives de son titulaire». 
la famille dans l'attribution de la charge curatélaire ou tutélaire ${ }^{35}$.

Ensuite, le majeur protégé dispose toujours d'une marge d'autonomie dans sa vie quotidienne. Il est d'usage de laisser à la personne majeure même soumise à une mesure d'incapacité d'exercice générale, de passer seule les actes de la vie courante ${ }^{36}$. La difficulté n'est pas de conclure des petits contrats de la vie quotidienne mais de disposer des fonds nécessaires à l'ambition de cette autonomie réduite. Le majeur protégé dispose alors d'une enveloppe de liquidités qui lui est remise par son tuteur à intervalles réguliers pour faire face à ses dépenses de la vie courante. La loi du 5 mars 2007 a renforcé l'idée d'autonomie qu'elle fonde sur le principe de respect de la dignité humaine ${ }^{37}$. L'article $498 \mathrm{du}$ Code civil a inversé le principe de l'épargne et l'exception de la libre consommation. Dorénavant, il ne revient plus au majeur en tutelle de gérer seul les sommes que le tuteur veut bien lui remettre. La loi oblige le juge ou le conseil de famille à «arrête $[\mathrm{r}]$ le budget ${ }^{3^{8}}$ en déterminant, selon la situation du majeur protégé, ses besoins personnels et ses charges patrimoniales, les sommes qui sont nécessaires à la gestion de son patrimoine et à l'entretien de ses biens. Le reste devra être remis au majeur en tutelle ${ }^{39}$. La loi prévoit aussi que le juge ou le conseil de famille puisse décider, à intervalles réguliers, de placer des fonds du majeur protégé sur des comptes d'épargne ${ }^{40}$.

En pratique, l'autonomie du majeur protégé a posé la question des bons d'achat. Cette technique de l'achat quasi affecté permettait aux associations tutélaires d'obtenir de centrales d'achat qu'elles fassent crédit au majeur protégé. Celui-ci pouvait engager seul toutes les dépenses qu'il souhaitait jusqu'au plafond fixé par le bon d'achat, et pendant une courte durée parfois renouvelable. La technique des bons d'achat permettait ainsi d'exclure l'acquisition de boissons alcooliques. Bien sûr, rien n'empêchait un majeur protégé de contourner cette contrainte en s'arrangeant avec un ami majeur non protégé pour procéder à des échanges. La technique des bons d'achat, à laquelle restent attachés certains majeurs protégés qui y voient un moyen sécurisant, tombe en désuétude dans les associations tutélaires car elle stigmatise la population des majeurs protégés et réduit leur autonomie.

En somme, la protection des biens du majeur protégé ne dépend pas d'un tuteur qui dispose de tous les pouvoirs.
La loi recherche un équilibre entre l'attribution d'un pouvoir de gestion au tuteur ou au curateur en curatelle renforcée, d'une part, l'attribution du pouvoir de contrôle au juge des tutelles alerté le cas échéant par le greffier en chef qui doit vérifier les comptes de gestion, d'autre part, et l'autonomie du majeur protégé, de troisième part. Nul ne prétend qu'en pratique cet idéal dosage se réalise dans la facilité et que les malades mentaux sont tous satisfaits de leur régime de protection. La loi elle-même rend subsidiaire les mesures de protection juridique ${ }^{41}$, sans abandonner alors le malade mental à son triste sort.

\section{B. Tournée vers le passé, la protection rétroactive des malades mentaux}

\section{Insania omnia corrumpit}

La loi du 5 mars 2007 a fait précéder les dispositions communes aux majeurs protégés par des règles indépendantes des mesures de protection. Introduites dans le Code civil par la loi du 3 janvier 1968 aux articles 489 et 489-1, ces règles siègent aujourd'hui aux articles 414-1 et 414-2 du Code civil. Toute personne qui a conclu un contrat sous l'empire d'un trouble mental peut saisir le juge de droit commun d'une demande en annulation. Impérative, la nullité est relative: l'action n'appartient qu'à l'intéressé, à l'instar des vices du consentement dont se rapproche le défaut total de consentement ${ }^{42}$. À sa mort, l'action en nullité est transmise à ses héritiers qui pourront agir à sa place. Mais deux difficultés se présentent toujours. La première est liée à la preuve de l'insanité. La seconde est liée au délai pour agir.

\section{La preuve du trouble mental}

La charge de la preuve du trouble mental incombe toujours au demandeur à l'action. Lorsque l'action est engagée par l'intéressé, de son vivant, la preuve du trouble mental peut être établie par tous moyens. Le juge raisonne alors par présomptions, comme le lui permet l'article 1353 du Code civil, car la preuve d'un fait psychologique est toujours indirecte ${ }^{43}$. À partir d'un faisceau d'indices constitués de faits tangibles établissant que l'intéressé souffrait d'une

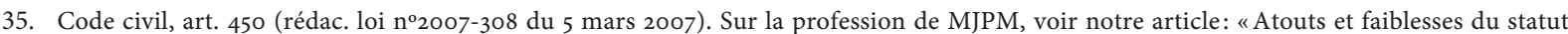
professionnel de mandataire judiciaire à la protection des majeurs», Droit de la famille, 2012, étude n 13, p. 16-21 (dossier: L. Mauger-Vielpeau, G. Raoul-Cormeil, «MJPM, état des lieux d'un statut professionnel et perspectives d'avenir », actes du colloque de la Faculté de droit de l'université de Caen Basse-Normandie, le 19 octobre 2012).

36. Pour un essai de définition, voir J.-C. Montanier, «Les actes de la vie courante en matière d'incapacités », La semaine juridique, éd. G, 1982, I, 3076.

37. Code civil, art. 415, al. 2 (rédac. loi n 2007-308 du 5 mars 2007).

38. Code civil, art. 500, al. 1 (rédac. loi $\mathrm{n}^{\circ} 2007-308$ du 5 mars 2007).

39. Code civil, art. 498, al. 1 (rédac. loi nº 2007-308 du 5 mars 2007).

40. Code civil, art. 501, al. 1 (rédac. loi no 2007-308 du 5 mars 2007).

41. Code civil, art. 428, al. 1 (rédac. loi no $2007-308$ du 5 mars 2007).

42. Cass., $1^{\mathrm{re}}$ civ., 11 janvier 2005, $\mathrm{n}^{\circ}$ 01-13.133, Bulletin civil I, $\mathrm{n}^{\circ}$ 23; Dalloz, 2005, Jur., p. 1207, note A.-L. Thomas-Raynaud; Droit de la famille, 2005, comm. n 63, note B. Beignier; Revue Lamy droit civil, mai 2005/16, nº67, p. 44, note C. Bernard-Xémard.

43. Sur la démonstration, voir notre thèse, La mauvaise foi dans les relations de droit privé, université de Caen Basse-Normandie, 2002 (dactyl.), spéc. n 467, p. 498. 
fragilité mentale chronique ${ }^{44}$ ou suivait un traitement médicamenteux qui a obscurci son jugement ${ }^{45}$, à des moments antérieurs ou postérieurs à la manifestation de son consentement, le juge accepte de se forger la conviction que l'intéressé n'était pas sain d'esprit lorsqu'il a conclu le contrat litigieux. La preuve par expertise médicale de l'influence d'un traitement médicamenteux ou d'une maladie chronique est souvent nécessaire ${ }^{46}$. Ainsi en est-il à l'égard d'une personne qui aura conclu un contrat contraire à son intérêt à un moment où il traversait une courte période d'insanité. La procédure des articles 414-1 et 414-2 du Code civil peut aussi intéresser l'entourage de malades mentaux qui ne font pas encore l'objet d'une mesure de protection juridique. Soit parce qu'il s'agit de personnes mariées dont le conjoint gère seul les richesses du ménage grâce aux mécanismes du droit des régimes matrimoniaux ${ }^{47}$. Soit parce qu'il s'agit de jeunes personnes majeures atteintes d'un handicap congénital à l'égard desquelles leurs père et mère n'ont pas encore jugé nécessaire de saisir le juge des tutelles aux fins de les placer sous un régime de protection. En raison de leur pleine capacité juridique, ces personnes majeures, malades mentales, qui ont souscrit des contrats contraires à leurs intérêts, trouveront une échappatoire à leur engagement dans la seule nullité pour insanité.

Une telle action en nullité pourra encore être engagée par les héritiers de l'intéressé. Mais l'article 414-2 du Code civil réduit la possibilité de rapporter la preuve par tous moyens de l'insanité du contractant aux seules libéralités, c'est-à-dire suivant l'article 893 du Code civil aux donations et aux testaments ${ }^{48}$. Pour les actes à titre onéreux conclus sous l'empire d'un trouble mental, les héritiers pourront prouver l'existence de ce trouble dans l'un des trois cas d'ouverture suivants: 1) si l'acte porte en lui-même la preuve d'un trouble mental; 2) si l'acte a été conclu à une époque où l'intéressé était placé sous sauvegarde de justice ou sous la protection d'un mandat d'inaptitude future qui avait pris effet;3) si l'intéressé a fait l'objet d'une requête devant le juge des tutelles aux fins d'ouverture d'une mesure de protection juridique qui est devenue caduque par son décès ${ }^{49}$. Cette restriction à la preuve par tous moyens de l'insanité a fait l'objet d'une question prioritaire de constitutionnalité (QPC) que la Cour de cassation a jugé sérieuse ${ }^{50}$. Mais le Conseil constitutionnel a maintenu les textes du Code civil au motif que le législateur ne compromet pas le droit des héritiers d'exercer un recours effectif devant une juridiction ${ }^{51}$. Les restrictions au droit d'agir sont le fruit d'un équilibre entre les intérêts des héritiers et la sécurité juridique des tiers. Le Conseil constitutionnel a été sensible au caractère incitatif que la loi a conféré à l'article 414-2, alinéa 2 du Code civil. La famille d'une personne vulnérable devait faire le nécessaire pour pourvoir à la défense de son intérêt. Si elle n'y parvenait pas, elle devait saisir le juge des tutelles aux fins de le faire bénéficier d'une mesure de protection. Dans le cas contraire, la difficulté d'engager une action quasi successorale sanctionne la négligence des héritiers qui n'ont pas fait le nécessaire du vivant de l'intéressé pour le placer sous la protection d'une mesure de curatelle ou de tutelle.

\section{Le délai pour agir en nullité}

La seconde difficulté est liée au délai pour agir en nullité. Si l'on s'en tient à la lettre de l'article 1304 du Code civil auquel renvoie l'article 414-1 in fine, la durée du délai est incertaine car le délai de cinq ans connaît trois points de départ. Entre la formation du contrat et le décès du contractant qui ouvre la succession, ce texte vise aussi la date à laquelle le majeur protégé peut refaire seul, valablement, le contrat conclu sous l'empire d'un trouble

44. Voir par exemple Cass., $\mathrm{1}^{\mathrm{re}}$ civ., $\mathrm{1}^{\mathrm{er}}$ juillet 2009, $\mathrm{n}^{\mathrm{o}} \mathrm{0} 8-13.518$, Bulletin civil $\mathrm{I}, \mathrm{n}^{\mathrm{o}} 150$; L'actualité juridique. Famille, 2009, p. 402, obs. L. PécautRivolier; Dalloz, 2009, Jur., p. 2660, note G. Raoul-Cormeil; Defrénois, 2009, p. 2336, obs. É. Savaux; Droit de la famille, 2009, comm. n 116, note I. Maria; Revue trimestrielle de droit civil, 2009, p. 507, obs. J. Hauser.

45. Voir par exemple Cass., $1^{\text {re }}$ civ., 20 octobre 2010, $\mathrm{n}^{\circ}$ 09-13.635, Bulletin civil $1, \mathrm{n}^{\mathrm{o}} 209$; L'actualité juridique. Famille, 2010, p. 496, obs. T. Verheyde; Dalloz, 2011, Jur., p. 50, note G. Raoul-Cormeil; Droit de la famille, 2010, comm. n 191, note I. Maria; Revue des contrats, 2011, p. 415, note É. Savaux, et p. 519, note P. Brun; Revue trimestrielle de droit civil, 2011, p. 103, obs. J. Hauser.

46. Dans le cas où l'action en nullité pour trouble mental est engagée par le représentant du majeur protégé, des juges ont accepté de présumer l'insanité du contractant à partir du certificat médical circonstancié pourtant établi dans les trois mois précédant l'ouverture de la mesure.

47. Voir ainsi M. Culioli, «La maladie d'un époux. Idéalisme et réalisme en droit matrimonial français », Revue trimestrielle de droit civil, 1968, p. 253-285; D. Langé, "Le conjoint de l'aliéné», Revue trimestrielle de droit civil, 1984, p. 33-68; V. Larribau-Terneyre, "Le régime matrimonial de l'incapable», La semaine juridique, éd. N, 1999, p. 843-849; J.-F. Sagaut, «Empêchement ou impéritie des époux: les solutions du droit des régimes matrimoniaux", L'actualité juridique. Famille, 2003, p. 124-129; C. Philippe, "Régimes matrimoniaux et altération des facultés mentales", Droit de la famille, 2006, étude nº 24, p. 33-36; G. Raoul-Cormeil, «Le conjoint de la personne vulnérable (l'articulation du système matrimonial et du système tutélaire)", Defrénois, 2008, art. 38791, p. 1303-1319.

48. La jurisprudence traite la souscription d'un contrat d'assurance sur la vie ou la modification de la clause bénéficiaire comme un acte à titre

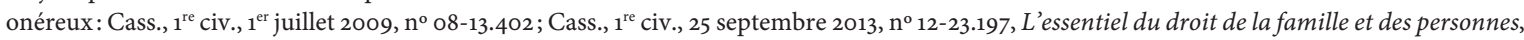
décembre 2013, n 164 , p. 2, obs. G. Raoul-Cormeil.

49. Voir, par exemple, à propos de la nullité d'un mariage obtenue à titre posthume par le frère et la sœur du défunt époux faute de consentement lucide: Cass., $1^{\text {re }}$ civ., 4 mai 2011, $\mathrm{n}^{\circ}$ 09-68983, Droit de la famille, 2011, comm. ${ }^{\circ}{ }_{145}$, note V. Larribau-Terneyre; Revue trimestrielle de droit civil, 2011, p. 516, obs. J. Hauser; Dalloz, 2011, Jur., p. 2387, note G. Raoul-Cormeil.

50. Cass., $1^{\text {re }}$ civ., 7 novembre 2012, $\mathrm{n}^{\circ}$ 12-40.068, Droit de la famille, 2013, comm. $\mathrm{n}^{\circ} 12$, note I. Maria; Revue trimestrielle de droit civil, 2013, p. 87, obs. J. Hauser.

51. CC, 17 janvier 2013, n 2012-288 QPC, Droit de la famille, 2013, comm. no 46, note I. Maria; L'essentiel du droit de la famille et des personnes, février 2013, nº 017 , p. 1, obs. A. Batteur qui critique la décision du Conseil constitutionnel lorsqu'il évoque la portée de l'abus de faiblesse dans la catégorie des vices du consentement sans montrer la difficulté d'établir devant un tribunal correctionnel la réalité de l'infraction. 
mental. Mais à l'égard d'une personne majeure qui souffre d'un handicap mental congénital et qui ne bénéficie pas encore d'une mesure de protection juridique, le temps jouera contre le contractant inconscient de l'imprudence de son acte. C'est pourquoi la Cour de cassation a ajouté un quatrième point de départ à la prescription quinquennale, en s'inspirant d'un adage latin: contra non valentem agere non currit praecriptio. Le délai pour agir en nullité ne court pas à l'encontre d'une personne qui ne peut pas agir tant qu'elle est sous l'empire d'un trouble mental ${ }^{52}$. L'ouverture de la tutelle au profit du malade mental fera alors courir le délai de la prescription extinctive à l'encontre du tuteur. Mais en cas d'inaction du tuteur, le tutélaire profitera encore du report du point de départ au jour où la mesure de tutelle s'éteindra, comme le lui permet la règle posée à l'article 2252 du Code civil, déplacée à l'article 2234 par la loi $\mathrm{n}^{\circ}$ 2008-561 du 17 juin 2008.

Tournée vers le passé lorsqu'elle est assurée par un texte qui fonde la nullité d'un contrat conclu par une personne insane, la protection des biens de la personne qui souffre d'une maladie mentale se renforce donc avec l'ouverture d'une mesure qui diminue sa pleine capacité juridique. La loi de 2007 a eu le mérite de fixer à deux ans précédant la publicité de la mesure ${ }^{53}$ la période suspecte pendant laquelle les actes souscrits par le curatélaire ou le tutélaire pourraient être contestés devant le juge de droit commun. Ainsi commandée par les circonstances ${ }^{54}$, la saisine du juge des tutelles ne débouche cependant plus aussi rapidement qu'autrefois sur l'ouverture d'une mesure de protection. La suppression de la saisine d'office par la loi du 5 mars 2007 expose certains malades mentaux à des dangers qui exigeraient une réaction immédiate de l'institution judiciaire. Or, la loi de 2007 n'a pas instauré de procédure d'urgence qui seule permettrait de traiter avec efficacité certaines difficultés auxquelles sont confrontés des majeurs à protéger. Il suffit de songer au conjoint d'un malade mental qui viderait tous les comptes bancaires pour vivre une passion amoureuse à l'étranger avec son amant pour réaliser la gravité de la situation dans laquelle serait plongée cette personne vulnérable. En l'état actuel des textes en vigueur, le juge des tutelles ne pourrait rien entreprendre s'il n'est pas valablement saisi. Le déclin de la protection des biens du majeur à protéger se justifie ici par un renforcement de la protection procédurale du sujet à protéger.

\section{La protection de la personne du malade mental}

La loi du 5 mars 2007 a consacré la jurisprudence qui a étendu la protection des biens du majeur protégé au gouvernement de sa personne. Sitôt ouverte, cette perspective appelait des nuances ${ }^{55}$. La protection de la personne passe d'abord par le respect de ses droits fondamentaux au nombre desquels figurent le respect de la parole en justice et le respect du corps humain, deux principes qui traduisent la protection du malade mental au cours de la procédure d'ouverture de la mesure (A). La protection de la personne se vérifie ensuite par l'exposé d'un régime juridique aussi riche que complexe puisque la loi de 2007 est descendue dans le détail de la protection de l'intimité individuelle (B).

\section{A. La protection au cours de l'ouverture de la mesure}

\section{L'institutionnalisation d'un médecin habilité}

Pour schématiser, le système tutélaire pourrait être représenté par un triangle aux sommets desquels figureraient la famille du majeur à protéger, l'autorité judiciaire et le savoir médical, l'intérêt du sujet vulnérable étant au centre de ce dispositif. Cet agencement résulte d'un arbitrage entre de la distance et de la proximité. La loi invite les familles à saisir le juge des tutelles lorsque ses membres vulnérables ne parviennent plus à pourvoir à la défense de leur intérêt ${ }^{56}$. Contrebalançant avec cette proximité, la distance avec l'intéressé résulte d'un examen médical approfondi réalisé non pas par le médecin traitant mais par un médecin choisi sur la liste du procureur de la République. Le certificat médical circonstancié que ce médecin habilité a rédigé est un document essentiel puisque le juge des tutelles y découvre le besoin de protection ${ }^{57}$ et, en conséquence, détermine la nature de la mesure de protection. Ce duo instauré par la loi du 3 janvier 1968 entre le médecin dit spécialiste et le juge des tutelles marquait un équilibre des forces et des pouvoirs entre le domaine judiciaire et le domaine médical. La loi du 5 mars 2007 a procédé à certains ajustements d'ordre technique qui ont pour effet de renforcer et de diversifier les missions du

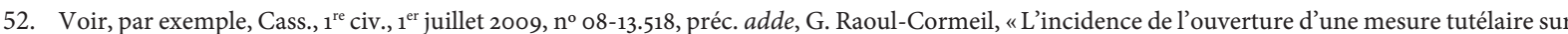
le point de départ de la prescription de l'action en nullité d'un contrat conclu sous l'empire d'un trouble mental ", Gazette du palais, n²59-260, 16-17 septembre 2009, p. 5-9.

53. Est inscrite en marge de l'acte de naissance de l'intéressé la mention «RC» suivie d'un numéro. Cette indication manifeste l'existence d'une mesure de protection juridique, sans préciser s'il s'agit d'une curatelle ou d'une tutelle. Sur le mécanisme régi par l'article 444 du Code civil et ses limites, voir D. Noguéro, «La publicité des mesures de protection des majeurs (Ouverture, vie et fin des mesures) », in Mélanges en l'honneur du professeur Jean Hauser, G. Wicker (dir.), Paris, LexisNexis - Dalloz, 2012, p. 467-533.

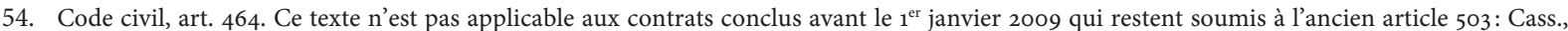
$1^{\text {re }}$ civ., 12 juin 2013, no 12-15.688, L'actualité juridique. Famille, 2013, p. 507, obs. G. Raoul-Cormeil; Dalloz, 2013, Jur., p. 1875, note D. Noguéro et A. Marais; Revue trimestrielle de droit civil, 2013, p. 577, obs. J. Hauser.

55. Code civil, art. 457-1 à 463 .

56. Code civil, art. 415, al. 4, 425 et 430 , combinés.

57. Voir le témoignage de A. Gauci-Scotté, «Le secret médical en matière de tutelle des personnes majeures », Revue générale de droit médical, juin $2012, n^{\circ} 43$, p. $65-72$ 
médecin des tutelles. Non plus réservée aux spécialistes que sont les psychiatres ou les gériatres, la liste est dorénavant ouverte aux généralistes qui en font la demande au parquet. Ces médecins sont agréés ou habilités par le procureur de la République après consultation du préfet, "c'est-à-dire, en fait, du médecin inspecteur de la santé ou du directeur départemental de l'action sanitaire et sociale $»^{58}$. Le statut de ce médecin et les missions qui sont les siennes dans la loi du 5 mars 2007 contribuent à la protection des personnes à protéger.

\section{Le statut du médecin habilité}

Les médecins habilités ne sont pas des experts, pourvu qu'on prête attention à la source de leur mission, au coût de l'examen médical et à la nature juridique du certificat médical. Alors que l'expert est toujours désigné par le juge pour éclairer le tribunal ou la cour, sur une liste établie par le procureur général près la cour d'appel ou près la Cour de cassation ${ }^{59}$, le médecin de l'article 431 du Code civil est choisi sur une liste établie par le procureur de la République, soit par le sujet ou le requérant lorsqu'il s'agit d'ouvrir une mesure, soit par la personne mandatée pour l'exécuter, le requérant, le ministère public ou le juge lorsqu'il s'agit d'un renouvellement. En conséquence, la personne qui a choisi ce médecin habilité est donc libre de faire appel à un autre médecin agréé si, ayant eu connaissance - à tort ou à raison ${ }^{60}$ - du contenu de son certificat, elle le jugeait insuffisant ou insatisfaisant. Ensuite, le coût d'un examen médical donnant lieu au certificat de l'article 431 du Code civil est de 160 euros $^{61}$, ce qui est bien moindre que le coût d'une expertise médicale. Le premier est fixé par décret, le second est déterminé par le juge qui désigne l'expert. Enfin, les certificats ou avis que doivent délivrer les médecins habilités n'obéissent pas au régime des mesures d'instruction ${ }^{62}$. Exigés par la loi, les certificats ou avis conditionnent la validité de la mesure ou de la décision du juge.

\section{Les missions du médecin habilité}

L'office du médecin habilité n'est cependant pas le même suivant qu'il est chargé de rédiger un certificat médical circonstancié ou de donner son avis. En premier lieu, la loi du 5 mars 2007 a réglementé le contenu du certificat médical circonstancié. Défini à l'article 1219 du Code de procédure civile ${ }^{63}$, ce document médical est devenu une pièce maîtresse de la procédure ${ }^{64}$, sans laquelle le juge des tutelles ne peut ouvrir une mesure de protection juridique $^{65}$. À suivre la loi à la lettre, ce certificat médical ne serait exigé que pour l'ouverture et l'aggravation de la mesure ${ }^{66}$, mais les juges des tutelles ont eu tendance à l'exiger systématiquement. En second lieu, la loi du 5 mars 2007 sollicite le médecin habilité pour qu'il donne trois avis différents. Tout d'abord, la loi invite ainsi le médecin habilité à délivrer, en cas de besoin, un avis de non-audition ${ }^{67}$. Les juges des tutelles doivent être en possession de cet avis - présent dans le certificat médical circonstancié ou établi dans un acte séparé - pour qu'ils soient valablement dispensés de solliciter la parole de l'intéressé. Les juges des tutelles observent ces avis de non-audition lorsque le médecin estime que l'audition de l'intéressé aurait pour effet de le perturber et d'aggraver son état de santé. Les juges sont néanmoins libres de ne pas suivre l'avis du médecin qui a simplement jugé inutile l'audition au regard de la gravité de l'altération des facultés mentales du sujet ${ }^{68}$. Ensuite, la loi de 2007 sollicite le médecin habilité à donner son avis lorsque le

58. J. Massip, Tutelle des mineurs..., $\mathrm{n}^{\circ} 283$.

59. R. Perrot, Institutions judiciaires, $15^{\mathrm{e}}$ éd., Paris, Montchrestien (Domat droit privé), 2012, nº 483, spéc. p. 370.

60. Les certificats médicaux circonstanciés sont rédigés à la seule destination du ministère public et des juges des tutelles. Dans le contexte d'un renouvellement, seul le tuteur d'un majeur peut en connaître le contenu car le secret médical ne s'impose pas à lui.

61. Art. R. 217-1 du Code de procédure pénale.

62. Sur lesquelles, voir J. Héron, Droit judiciaire privé, $5^{\mathrm{e}}$ éd. par T. Le Bars, Paris, Montchrestien, 2012, $\mathrm{n}^{\circ} 1128$.

63. «Le certificat médical circonstancié prévu par l'article 431 du code civil: $1^{\circ}$ Décrit avec précision l'altération des facultés du majeur à protéger ou protégé; $2^{\circ}$ Donne au juge tout élément d'information sur l'évolution prévisible de cette altération; $3^{\circ}$ Précise les conséquences de cette altération sur la nécessité d'une assistance ou d'une représentation du majeur dans les actes de la vie civile, tant patrimoniaux qu'à caractère personnel, ainsi que sur l'exercice de son droit de vote. Le certificat indique si l'audition du majeur est de nature à porter atteinte à sa santé ou si celui-ci est hors d'état d'exprimer sa volonté. Le certificat est remis par le médecin au requérant sous pli cacheté, à l'attention exclusive du procureur de la République ou du juge des tutelles».

64. Voir l'étude de F. Fresnel, «Le certificat médical, une pièce maîtresse de la mesure de protection des majeurs », Dalloz, 2010, Point de vue, p. 2656. Adde, D. Noguéro, «Le certificat médical pour l'ouverture des mesures de protection des majeurs», Revue de la recherche juridique, ${ }^{\circ} 3$, 2011, p. 1227-1252.

65. Et ce quelle que soit la nature de la mesure: sauvegarde de justice, curatelle ou tutelle. L'activation d'un mandat de protection future est aussi subordonnée à la présentation par le mandataire au greffier du tribunal d'instance d'un certificat médical circonstancié concernant la personne du mandatant (art. 481, al. 2 du Code civil).

66. Code civil, art. 442 in fine. Comment le juge des tutelles pourrait-il en effet être libre de renouveler la mesure s'il ne pouvait pas l'aggraver? Sa liberté de décision suppose qu'il soit en possession d'un certificat médical circonstancié rédigé au regard de l'état de santé actualisé du majeur protégé. Le caractère systématique de la demande de ce certificat médical pèche néanmoins par excès car une mesure de tutelle complète ne peut pas être aggravée. Cette formalité paraît donc ici inutile. Ce qui est gênant lorsque le majeur protégé n'a pas les moyens de s'acquitter des 160 euros exigés, puisque c'est lui qui supporte le coût du renouvellement de sa mesure.

67. Code civil, art. 432, al. 2. Sur lequel, voir F. Fresnel, «Le contentieux de l'ouverture d'une mesure de protection", in Nouveau droit des majeurs protégés..., p. 25-36, spéc. p. 30.

68. Des juges se déplacent ainsi dans les établissements d'hébergement pour les personnes âgées dépendantes (EHPAD) pour mettre un visage sur une requête et constater par eux-mêmes leur besoin de protection. C'est souvent l'occasion pour ces magistrats de vérifier in situ la manière dont sont traitées les personnes très vulnérables et d'exercer ainsi leur mission de surveillance générale (cf. Code civil, art. 417). 
tuteur ou le curateur envisage la vente du logement de l'intéressé et son placement corrélatif dans un établissement d'hébergement collectif ${ }^{69}$. Enfin, la loi de 2007 interdit au juge des tutelles de fixer la durée de la mesure pour un délai de plus de cinq ans si sa décision n'est pas prise sur "avis conforme ${ }^{70}$ du médecin habilité. Dans cet avis de longue mesure, le médecin habilité doit expliquer les raisons médicales pour lesquelles il n'apparaît pas nécessaire de réviser la mesure dans les cinq prochaines années. La stabilité de la maladie mentale et son caractère incurable au regard des données actuelles de la science sont les arguments attendus. Toutes ces règles ont pour but de protéger les personnes vulnérables.

\section{Le problème du refus du malade mental de se laisser examiner par le médecin}

En pratique, les requêtes en ouverture d'une mesure de protection sont présentées par les membres de la famille du majeur à protéger, voire le ministère public, rarement par l'intéressé même si la loi le lui permet ${ }^{71}$. Dans tous les cas où c'est un tiers qui a pris l'initiative de la requête, l'exigence d'un certificat médical circonstancié est susceptible de se heurter au refus de l'intéressé de se soumettre à l'examen médical. Sous l'empire de la loi du 3 janvier 1968, la curatelle ou la tutelle ne pouvait être ouverte pour altération des facultés mentales si celle-ci n'était pas médicalement constatée par un médecin spécialiste ${ }^{72}$. Mais la Cour de cassation avait élevé un tempérament à cette exigence. Elle avait jugé que la personne protégée «n'est pas fondée à se prévaloir de l'absence de constatation médicale de l'altération de ses facultés lorsque, par son propre fait, elle a rendu cette constatation impossible en se refusant à tout examen ${ }^{73}$. Au lendemain de l'entrée en vigueur de la loi du 5 mars 2007 , la question s'est posée de savoir si la Cour de cassation pouvait être fidèle à sa jurisprudence ou si elle devait la faire évoluer. Les travaux préparatoires militaient pour le statu quo car les rapporteurs n'avaient pas manifesté la volonté de briser la jurisprudence ${ }^{74}$. Militait en revanche pour un durcissement prétorien le principe bioéthique selon lequel le corps humain est inviolable ${ }^{75}$. Ainsi le juge des tutelles ne devait pas se montrer insensible au refus du patient de se laisser examiner. De surcroît, l'introduction d'une sanction procédurale à l'absence de certificat médical circonstancié paraît constituer un principe absolu: la requête doit être déclarée irrecevable par le juge des tutelles dès lors qu'elle n'est pas accompagnée d'un certificat médical circonstancié ${ }^{6}$. Les juges du fond ont donc cru possible d'accueillir la requête accompagnée d'un procès-verbal de carence du médecin habilité 77 . Mais ce jugement d'appel a été cassé au visa de l'article 431 du Code civil ${ }^{78}$, dont la lettre constitue le seul motif de la violation de la loi. La cassation disciplinaire de ce jugement n'a donc pas éclairé les juges du fond sur la conduite à tenir en cas de refus obstiné du majeur vulnérable de se laisser examiner. Attentive à la formulation de l'article 415, alinéa 1 du Code civil aux termes duquel «les personnes majeures reçoivent la protection de leur personne et de leurs biens [...]», la doctrine ${ }^{79}$ a encouragé les juges du fond à résister, en forgeant le concept de certificat médical circonstancié de carence qui doit être aussi bien circonstancié sinon mieux que si le médecin avait pu examiner le patient. Comme tout certificat médical circonstancié, ce document devra constater l'altération des facultés mentales de l'intéressé, décrire la pathologie et ses conséquences sur les actes importants de la vie civile ${ }^{80}$. Une telle constatation devra donc être établie par un médecin habilité qui consultera le dossier médical des médecins traitants de l'intéressé. L'avis du médecin traitant pourra aussi être sollicité ${ }^{81}$. Le secret

69. Code civil, art. 426, al. 3 . L'article $1^{\text {er }}$ du projet de loi relatif à la modernisation et à la simplification du droit et des procédures dans les domaines de la justice et des affaires intérieures (Sénat, 27 novembre 2013, $\mathrm{n}^{\circ} 175$ ) prévoit cependant de confier cet avis au médecin traitant.

70. Cass., $1^{\text {re }}$ civ., 10 octobre 2012, no $11-14.441$, L'actualité juridique. Famille, 2012, p. 619, obs. T. Verheyde; Droit de la famille, 2012 , comm. $\mathrm{n}^{\circ}$ 184, note I. Maria; Dalloz, 2012, Jur., p. 2723, note G. Raoul-Cormeil; Revue trimestrielle de droit civil, 2013, p. 92, obs. J. Hauser.

71. Code civil, art. 430.

72. Code civil, anc. art. 490, al. 3 (rédac. loi n 68-5 du 3 janvier 1968): «L'altération des facultés mentales ou corporelles doit être médicalement établie». Ce texte doit être combiné à l'ancien article 493-1 pour la tutelle ou à l'ancien article 508 pour la curatelle. Seule la curatelle pour prodigalité - abrogée par la loi du 5 mars 2007 - faisait exception à cette exigence; cf. Code civil, anc. art. 508-1. Voir, en ce sens, Cass., $1^{\text {re }}$ civ., 24 octobre 1995, nº 93-21.590, Dalloz, 1997, Jur., p. 22, note B. Fillion-Dufouleur; La semaine juridique, éd. G, 1996, II, 22698, note F. Kessler; Revue trimestrielle de droit civil, 1996, p. 132, obs. J. Hauser.

73. Cass., $\mathrm{1}^{\mathrm{re}}$ civ., 10 juillet $1984, \mathrm{n}^{\mathrm{o}} 83-10.653$, Dalloz, 1984, 547, note J. Massip.

74. H. de Richemont, Rapport fait au nom de la commission des lois constitutionnelles, de législation, du suffrage universel, du règlement et d'administration générale sur le projet de loi, adopté par l'Assemblée nationale, après déclaration d'urgence, portant réforme de la protection juridique des majeurs, $\mathrm{n}^{\circ} 212$, Sénat, 7 février 2007, p. 130.

75. Code civil, art. 16-1 (rédac. loi nº 94-653 du 29 juillet 1994).

76. Code civil, art. 431 (rédac. loi $\mathrm{n}^{\circ}$ 2007-308 du 5 mars 2007).

77. TGI Mont de Marsan, 8 octobre 2009, Dalloz, 2010, Jur., p. 2052, note T. Verheyde.

78. Cass., $1^{\text {re }}$ civ., 29 juin 2011, nº 10-21.879, L'actualité juridique. Famille, 2011, p. 431, obs. T. Verheyde; Dalloz, 2011, Panor., p. 2502, obs. J.-M. Plazy; Droit de la famille, 2011, comm. n 133, note I. Maria; La semaine juridique, éd. G, 2011, 987, note N. Peterka; L'essentiel du droit de la famille et des personnes, septembre 2011, n 130, p. 5, obs. G. Raoul-Cormeil; Revue trimestrielle de droit civil, 2011, p. 511, obs. J. Hauser.

79. J. Hauser, «Ouverture d'un régime de protection et certificat circonstancié», Revue trimestrielle de droit civil, 2011, p. 511; J. Massip, « De quelques problèmes posés par la protection juridique des majeurs», La semaine juridique, éd. N, 2011, étude n 1244; H. Fulchiron, «Peut-on protéger la personne contre elle-même?», La semaine juridique, éd. N, 2012, étude no 1196 ; H. Fulchiron, «Peut-on protéger la personne contre elle-même? Réflexions autour de l'affaire Bettencourt», in Mélanges en l'honneur de la professeure Françoise Dekeuwer-Défossez, M. Dupuis (dir.), Paris, Montchrestien-Lextenso, 2012, p. 117-145.

80. Code civil, art. 431 (rédac. loi $n^{\circ} 2007-308$ du 5 mars 2007).

81. Code de procédure civile, art. 1219 (rédac. décret $\mathrm{n}^{\circ}$ 2008-1276 du 5 décembre 2008). 
médical ne pourra pas être opposé à un médecin inscrit sur la liste du procureur de la République. Le secret médical ne sera pas violé car le destinataire de ce certificat médical est l'autorité judiciaire à l'égard de laquelle aucun secret professionnel ne peut être opposé. Par ailleurs, le médecin habilité devra expliquer les raisons pour lesquelles il n'a pu examiner l'intéressé. Il devra alors indiquer les moyens mis en œuvre pour le rencontrer et les échecs constatés, bref ce qui lui permet d'avoir la conviction que l'intéressé manifeste une obstination maladive de se laisser examiner. Le respect des droits fondamentaux de la personne à protéger ne doit pas se retourner contre elle. Une telle analyse découle de deux arrêts de cour d'appel ${ }^{82}$ qui peuvent être cités dans le sens d'une résistance modérée.

Ainsi le malade mental qui ferait une objection systématique n'échapperait pas à son placement en tutelle ou en curatelle renforcée. Il serait difficile pour le juge des tutelles de choisir la curatelle simple tant ce régime exige une relation de confiance entre le curatélaire et le curateur pour respecter la technique d'assistance requise pour la conclusion des actes de disposition. Les difficultés liées à la mise en œuvre de la condition d'assistance ${ }^{8_{3}}$ conduiraient sans doute le juge des tutelles à choisir la tutelle, quitte à prévoir que le tutélaire peut passer seul certains actes juridiques comme en curatelle simple ${ }^{84}$. La protection de la personne du malade mental ne se limite pas à l'ouverture de la mesure, elle se prolonge dans son fonctionnement.

\section{B. La protection au cours de la mise en œuvre de la mesure}

\section{La considération de la loi pour l'intimité du majeur protégé}

Consacrant la jurisprudence ${ }^{85}$ qui a étendu la protection des biens du majeur protégé à la protection de sa personne, la loi du 5 mars 2007 a brisé le silence de la loi de 1968, révélant la difficulté de protéger la personne sans l'associer aux décisions qui concernent son intimité ${ }^{86}$. Les techniques de la surveillance, de l'assistance et de la représentation ne cessent pas d'être pertinentes mais elles ne peuvent plus être associées à la sauvegarde de justice, à la curatelle et à la tutelle. Le respect des droits fondamentaux du majeur protégé signifie que le majeur protégé gouverne seul sa vie. La protection de la personne n'est rien d'autre, en principe, qu'une disposition de surveillance et d'alerte du juge des tutelles en cas d'aggravation de l'état de santé du majeur protégé. La technique de l'assistance n'est pas repoussée du domaine de la protection de la personne. Elle permet de respecter la personnalité et les choix du majeur protégé tout en obligeant un tiers à contrôler en amont la conformité de sa décision à son intérêt. La technique de l'assistance caractérise la catégorie des droits simplement personnels. Par contraste, la loi a voulu créer un sanctuaire du majeur protégé où son intimité ne peut être violée. La catégorie des droits strictement personnels abrite les décisions que seul le majeur protégé peut prendre, sans jamais pouvoir être assisté ni représenté. Le juge des tutelles a néanmoins le pouvoir d'ajuster les actes personnels qui ne font pas l'objet d'un régime spécifique. Il peut élever la prise de décision à l'assistance du curateur ou du tuteur. Il peut même élever la décision à la représentation du tuteur pourvu que cette décision apparaisse possible à être mise en œuvre. N'oublions pas que l'on ne peut pas, quoi qu'en dise la chanson, vivre par procuration...

\section{Les droits simplement personnels}

L'union et la désunion font l'objet d'un régime juridique de protection où le malade mental doit être associé à la décision sans pouvoir la prendre seul. Ainsi le mariage du curatélaire doit être autorisé par son curateur ${ }^{87}$. Le curateur peut déléguer cette mission au juge s'il éprouve des difficultés à se faire un avis sur l'intérêt du majeur protégé à se marier ${ }^{88}$. Le juge des tutelles sera donc toujours sollicité pour autoriser le mariage du tutélaire. Le mariage est une décision grave qui peut remettre en cause l'existence même de la mesure de protection juridique en vertu du principe de subsidiarité. La consultation de la famille du majeur protégé n'est plus obligatoire. L'appréciation de l'intérêt à se marier doit prendre en considération tous les effets du mariage, d'ordre personnel (fidélité, secours, assistance,

82. CA Douai, 11 janvier 2013, $n^{\circ} \mathrm{RG}$ 12/05941, L'actualité juridique. Famille, 2013, p. 136, obs. G. Raoul-Cormeil; Droit de la famille, 2013, comm. n 45 ( $2^{\mathrm{e}}$ esp.), note I. Maria; CA Paris, 8 janvier 2013, $\mathrm{n}^{\mathrm{o}} \mathrm{RG}$ 11/18841, Droit de la famille, 2013, comm. $\mathrm{n}^{\mathrm{o}} 45$ ( $1^{\mathrm{re}}$ esp.), note I. Maria.

83. Code civil, art. 467, al. 2 (rédac. loi nº $2007-308$ du 5 mars 2007).

84. Code civil, art. 473, al. 2 (rédac. loi no $2007-308$ du 5 mars 2007).

85. Cass., $1^{\text {re }}$ civ., 18 avril 1989, n 87-14.563, Bulletin civil I, $\mathrm{n}^{\circ} 156$; La semaine juridique, éd. G, 1989, II, 21467, note T. Fossier; G. Raoul-Cormeil, "La protection de la personne même du majeur protégé», in Les grandes décisions du droit des personnes et de la famille, A. Batteur (dir.), Paris, LGDJ-Lextenso, 2012, no 431 sq., p. 380-387.

86. Parmi une littérature déjà abondante, voir T. Fossier, «La réforme de la protection des majeurs... »; T. Fossier, «La protection de la personne, un droit flexible», Droit de la famille, 2007, étude no 15, p. 15-20; A. Batteur, «Majeurs protégés. Curatelle et tutelle. Effets personnels. Articles 457-1 à 463 ", Jurisclasseur Code civil, janvier 2009; P. Salvage-Gerest, «Les actes dont la nature implique le consentement strictement personnel du majeur en tutelle (Code civil, art. 458) : une catégorie à revoir d'urgence», Droit de la famille, 2009, étude n 17, p. 19-24; J. Massip, «Les règles applicables aux actes personnels et médicaux concernant un majeur en tutelle», Droit de la famille, 2010, étude nº 18, p. 18-19; D. Guével, «La protection des actes personnels et familiaux des majeurs vulnérables», Les petites affiches, n spécial, 4 novembre 2010, p. 30-34; J. Hauser, «Le majeur protégé, acteur familial», Droit de la famille, 2011, étude n 6, p. 28-33; S. Moracchini-Zeidenberg, "L'acte personnel de la personne vulnérable", Revue trimestrielle de droit civil, 2012, p. 21-32 ; F. Fresnel, «La protection de la personne: nouvelle mission des associations tutélaires », Juris Associations, $\mathrm{n}^{\circ} 20, \mathrm{n}^{\circ} 490$, décembre 2013, p. 24-27 (dossier: «Protection des majeurs: un bilan nuancé»).

87. Code civil, art. 460, al. 1 (rédac. loi no 2007-308 du 5 mars 2007).

88. Code civil, art. 460, al. 2 (rédac. loi nº2007-308 du 5 mars 2007). 
communauté de vie) et d'ordre patrimonial (contribution aux charges du mariage, solidarité des dettes ménagères, régime matrimonial). L'appréciation de l'intérêt nuptial par un tiers n'est pas une limite disproportionnée à la liberté nuptiale dès lors qu'il existe notamment des voies de recours. La QPC a été rejetée par le Conseil constitutionnel $^{89}$. Le divorce n'est pas une décision plus facile à prendre pour le juge des tutelles qui ne sera saisi dans la curatelle qu'en cas de désaccord entre le curateur et le curatélaire. L'effet de la curatelle et de la tutelle est surtout de fermer deux cas de divorce sur les quatre aujourd'hui en vigueur ${ }^{90}$. Seuls les divorces pour faute ou pour altération des facultés mentales sont autorisés. Mais les juges aux affaires familiales ne sont pas toujours très vigilants et homologuent parfois des conventions de divorce par consentement mutuel conclues par un majeur protégé ${ }^{91}$. La conclusion, la modification et la révocation du pacte civil de solidarité sont également soumises à la technique de l'assistance, même si la loi distingue le principe de la décision qui doit être prise par le seul majeur protégé et les modalités de mise en œuvre qui suscitent l'assistance ou la représentation du curateur ou du tuteur pour assurer l'efficacité de la décision, se soumettre aux obligations légales et aux conditions de publicités2 ${ }^{2}$ La santé du majeur protégé donne également matière à enrichir la catégorie des droits strictement personnels. Le médecin ne peut pas opposer le secret médical au tuteur d'un majeur protégé ${ }^{93}$ et doit l'associer au consentement du majeur protégé à l'acte médical ${ }^{94}$. Bien sûr, le médecin devra respecter le refus du patient, même sous tutelle, de consentir à un acte médical, à moins que le juge des tutelles n'autorise expressément, et exceptionnellement, le tuteur à représenter le tutélaire ${ }^{95}$. Le tuteur devra aussi saisir le juge des tutelles à chaque fois que l'acte médical portera atteinte à l'intégrité corporelle du majeur protégé ${ }^{96}$, ce qui est le cas en pratique en cas d'arrachage de toutes les dents, de l'amputation d'un membre ou de l'ablation d'un sein ${ }^{97}$. En revanche, l'arrachage d'une dent et une coloscopie ${ }^{98}$ ne sont pas subordonnés à l'autorisation du juge des tutelles. Des textes spéciaux obligent par ailleurs le tuteur mais aussi le curateur à saisir le juge des tutelles pour des actes médicaux exceptionnels tels que le don de cellules hématopoiétiques issues de la moelle osseuse ${ }^{99}$, la recherche biomédicale à des fins scientifiques ${ }^{100}$ et la stérilisation contraceptive ${ }^{101}$. Le rôle du tuteur en matière de santé devrait être décisif du choix de la tutelle en matière de protection des malades mentaux dont on pressent le besoin à ne pas être désemparé devant leur difficulté. L'assistance du tuteur en matière de santé est d'autant plus nécessaire que le malade mental est dans une situation de déni. La protection connaît toutefois des limites depuis que la loi reconnaît au majeur protégé des droits strictement personnels.

\section{Les droits strictement personnels}

Situé au cœur des effets personnels de la curatelle et de la tutelle, l'article $458 \mathrm{du}$ Code civil définit les actes juridiques que le majeur protégé doit conclure seul ${ }^{102}$ comme ceux «dont la nature implique un consentement strictement personnel». Si cette définition a du sens ${ }^{103}$, la référence à la nature inhérente à l'acte juridique renvoie à l'intimité de son auteur. Or, cette discrète évocation de la capacité naturelle du majeur protégé manifeste une mutation de la notion. Suivant une approche classique, la capacité naturelle était liée à la raison du sujet, dont l'intervalle de lucidité en est «la manifestation la plus éclatante ${ }^{104}$. Dans une approche renouvelée, la capacité naturelle est

89. CC, déc. n 2012-26o QPC du 29 juin 2012, M. Roger D., L'actualité juridique. Famille, 2012, p. 463, obs. T. Verheyde; Dalloz, 2012, Point de vue, p. 1899, obs. G. Raoul-Cormeil; Droit de la famille, 2012, comm. ${ }^{\circ}$ 148, note I. Maria; Revue trimestrielle de droit civil, 2012, p. 510, obs. J. Hauser. Adde, F. Fresnel, «La triade: le majeur protégé, la mesure de protection et le mariage. Paradis, purgatoire ou enfer? », L'actualité juridique. Famille, 2012, p. 250. Prise en matière de curatelle, cette décision a une portée plus grande; elle s'impose à la tutelle par un raisonnement $a$ fortiori. En outre, elle peut être transposée, à l'égard de ces deux mesures, pour la conclusion du pacte civil de solidarité (Code civil, art. 461 et 462 ).

90. Code civil, art. 249-4.

91. Un jugement du 19 juin 2012 du juge aux affaires familiales de Caen ( ${ }^{\circ}$ 12/01283, UDAF du Calvados, ès qualité de curatrice de $M^{\text {me }}$ J. épouse C. c. M. C. et $\mathrm{M}^{\mathrm{me}}$ J. épouse C., M. Le Bourvellec) rétracte un divorce par consentement mutuel prononcé au profit d'une majeure en curatelle, alors pourtant que la loi l'interdit formellement. Sur cette décision, voir L. Mauger-Vielpeau, «Le divorce par consentement mutuel du curatélaire», Gazette du palais, $\mathrm{n}^{\circ} 17,17$ janvier 2013, p. 12.

92. Pour un commentaire des articles 461 et 462 du Code civil, voir L. Mauger-Vielpeau, "Couple et majeur protégé», in Nouveau droit des majeurs protégés..., p. 243-258.

93. Code de la santé publique (CSP), art. L. 1111-2, al. 5.

94. CSP, art. L. 1111-6, al. 4 .

95. Code civil, art. 459, al. 2 in fine.

96. CSP, art. L. 459 , al. 3.

97. Sur cette jurisprudence non publiée des juges des tutelles, voir notre article, «Remèdes à l'éclatement du régime juridique des actes médicaux portant sur les majeurs protégés", in Nouveau droit des majeurs protégés..., p. 151-172.

98. TI Nice, ord. JT, 4 février 2009, Dalloz, 2009, Jur., p. 1397, note T. Verheyde; Droit de la famille, 2009, comm. nº 147, note L. Talarico; Revue trimestrielle de droit civil, 2010, p. 509, obs. J. Hauser. Adde, J. Massip, «Les règles applicables aux actes personnels et médicaux concernant un majeur en tutelle», Droit de la famille, 2010, étude n 18, p. 18, spéc. $n^{\circ} 7$.

99. CSP, art. L. 1241-4 (rédac. loi no $2004-800$ du 6 août 2004).

100. CSP, art. L. 1122-2 (rédac. loi nº 2004-806 du 9 août 2004). Ce texte n'a pas été modifié par la loi nº 2012-300 du 5 mars 2012 relative aux recherches impliquant la personne humaine, cf. JORF, $\mathrm{n}^{\circ} 56,6$ mars 2012, p. 4138.

101. CSP, art. L. 2123-2 (rédac. loi no 2001-588 du 4 juillet 2001).

102. Sans avoir besoin de solliciter l'assistance ou la représentation d'un tiers.

103. Contra F. Terré, D. Fenouillet, Droit civil: les personnes, $8^{\mathrm{e} e ́ d ., ~ P a r i s, ~ D a l l o z ~(P r e ́ c i s . ~ D r o i t ~ p r i v e ́), ~ 2012, ~ n º ~ 645, ~ p . ~} 685$

104. J.-M. Plazy, La personne de l'incapable, $\mathrm{n}^{\circ} 354$, p. 240 qui emploie l'expression médicale de période de rémission. 
fondée sur la dignité humaine ${ }^{105}$. Réduite à la catégorie des actes éminemment personnels, la capacité naturelle est le sanctuaire de l'autonomie du majeur protégé. Synonyme d'exclusivité, elle est vouée à cette alternative: la liberté pour la personne protégée saine d'esprit ou l'impossibilité pour elle de jouir de ses droits en cas d'insanité, car il faut être sain d'esprit pour conclure un acte juridique ${ }^{106}$. Brisant un précieux silence séculaire, source de riches opinions doctrinales ${ }^{107}$, la règle de l'article 458 du Code civil aurait gagné en force à rester dans un non-dit baigné de droit naturel. Seules des circonstances délicates auraient dû contraindre la jurisprudence à dévoiler, au cas par cas, l'existence positive de cette capacité naturelle sans l'enfermer dans une définition bloquée et une liste imprudente.

Impérative, la liste dressée à l'alinéa 2 de l'article 458 du Code civil n'est pas exhaustive.

Sont réputés strictement personnels la déclaration de naissance d'un enfant, sa reconnaissance, les actes de l'autorité parentale relatifs à la personne d'un enfant, la déclaration du choix ou du changement de nom d'un enfant et le consentement donné à sa propre adoption ou à celle de son enfant.

Beaucoup d'auteurs ${ }^{108}$ ont critiqué la lettre de ce texte. Tout d'abord, la déclaration de naissance d'un enfant et le choix de son nom de famille ne se font qu'en mairie. Précisons que seules les mentions du nom de la mère et du nom du père marié auraient dû être subordonnées au consentement personnel des majeurs protégés, car la loi en fait des titres de filiation. La maladresse a consisté à faire fi du devoir de toute personne ayant assisté à l'accouchement de déclarer la naissance d'un nouveau-né, l'article $56 \mathrm{du}$ Code civil ne reconnaissant au père présent qu'une priorité de diligence.

Ensuite, la reconnaissance peut être manifestée devant tout officier public habilité à dresser un acte authentique. Aujourd'hui ouverte aux pères mariés ${ }^{109}$, la reconnaissance est un acte strictement personnel parce qu'elle porte l'aveu de son auteur d'avoir eu des relations sexuelles avec la mère de l'enfant pendant la période légale de conception.

Par ailleurs, la demande en changement de nom d'un enfant mineur se fait par le dépôt au parquet de la requête administrative adressée au garde des Sceaux.
Quant aux actes de l'autorité parentale, ils se manifestent facilement, sans le besoin de recourir au juge, et pas seulement devant l'administration scolaire ou hospitalière.

Enfin, le consentement donné par le majeur protégé à sa propre adoption ${ }^{110}$ ou à l'adoption de son enfant mineur est recueilli par le tribunal de grande instance. Cela étant, dans l'une ou l'autre de ces procédures le majeur protégé n'est pas le demandeur à la requête. Aussi avait-il été soutenu que la requête en adoption formée par le majeur protégé devrait, en ce qu'elle constitue une action en justice, échapper à l'article $458 \mathrm{du}$ Code civil. Et la recevabilité de cette requête aurait dû être soumise, suivant la nature de la mesure, à l'assistance du curateur ou la représentation du tuteur autorisé par le juge des tutelles.

En somme, aucune des sept prérogatives réservées au curatélaire ou au tutélaire ne doit être mise en œuvre par une décision de justice, de sorte que l'on pouvait penser que la loi du 5 mars 2007 n'avait pas consacré la capacité d'ester en justice. Le curatélaire devait être assisté de son curateur pour engager une action ou y défendre. De même, le tutélaire devait être représenté par son tuteur qui devait de surcroît solliciter l'autorisation du juge des tutelles pour les actions extrapatrimoniales.

Fondée sur les articles 468, alinéa 3 et 475 du Code civil, l'analyse a été récemment remise en cause par la Cour de cassation qui retranche dorénavant de la classification des actions en justice celles que le majeur protégé peut engager seul pour faire valoir ses droits strictement personnels. Audacieuse, cette jurisprudence a été prise à propos de la capacité juridique reconnue à une mère, placée en tutelle, d'interjeter appel de la décision du juge des enfants qui restreignait son droit de visite sur son enfant, un très jeune mineur ${ }^{111}$. En l'espèce, la position de la Cour de cassation était pleinement justifiée parce qu'il était excessif de priver une mère, fût-elle sous tutelle, de la faculté de contester la décision d'un juge qui ne lui permettait de voir son enfant qu'à raison d'une heure tous les quinze jours. L'extension de la catégorie des droits strictement personnels aux actions en justice relatives à la mise en œuvre de prérogatives parentales n'est peutêtre qu'un coup d'essai. Eu égard aux effets pervers que l'analyse procédurale des actions attitrées a révélés, la Cour de cassation sera sans doute conduite à donner un tour restrictif à l'évolution jurisprudentielle qu'elle a initiée.

105. Code civil, art. 415, al. 2. Adde, E. G. Sledziewski, «La dignité du sujet vulnérable», Droit de la famille, 2011, dossier nº 8 , p. $37-39$, spéc. nº 3, p. 38. Comp. F. Betaillole-Gonthier, La capacité naturelle, thèse de doctorat, université Bordeaux 4, 1999 (dactyl.).

106. Code civil, art. 414-1.

107. Voir les références précitées (n. 86).

108. Voir T. Fossier, «La réforme de la protection des majeurs. Guide de lecture de la loi du 5 mars 2007 », spéc. $n^{\circ}$ 19; A. Batteur, «Majeurs protégés. Curatelle et tutelle. Effets personnels... ", spéc. n 47 ; P. Salvage-Gerest, «Les actes dont la nature implique le consentement strictement personnel du majeur en tutelle (Code civil, art. 458): une catégorie à revoir d'urgence», Droit de la famille, 2009, étude n 17, p. 19-24; A. Batteur, «Le majeur protégé et l'enfant», in Nouveau droit des majeurs protégés..., p. 223-242.

109. Code civil, art. 316.

110. Voir, sur l'anticipation de l'application de la règle par la jurisprudence, Cass., $1^{\text {re }}$ civ., 8 octobre 2008, nº 07-16.094, Droit de la famille, 2008, comm. $\mathrm{n}^{\circ} 173$, note P. Murat; La semaine juridique, éd. G, 2008, II, 10012, note Y. Favier; Revue trimestrielle de droit civil, 2008, p. 655, obs. J. Hauser.

111. Cass., $1^{\text {re }}$ civ., 6 novembre 2013, $\mathrm{n}^{\circ}$ 12-23.766, Bulletin civil I; L'actualité juridique. Famille, 2013, p. 717, obs. T. Verheyde; La semaine juridique, éd. G, $\mathrm{n}^{\circ}$ 1-2, 2014, 14, note N. Peterka; Droit de la famille, 2014, comm. no 9, note I. Maria; Dalloz, 2014, Jur., p. 467, note G. Raoul-Cormeil; Revue trimestrielle de droit civil, 2014, p. 86, obs. J. Hauser. 


\section{Conclusion}

En définitive, la protection du malade mental est peutêtre plus grande lorsqu'il fait l'objet d'une mesure de protection juridique que lorsqu'il jouit de sa pleine capacité juridique. C'est un paradoxe aisément explicable. Le tuteur ou le curateur de la personne malade mentale doit garantir le respect de ses droits fondamentaux et de son intimité, notamment dans la mise en œuvre de ses droits strictement personnels. Le meilleur moyen de ne pas céder à l'angélisme est d'inviter les familles à honorer leur devoir de prendre en charge les difficultés de leur parent vulnérable car, malgré leurs efforts et le sens de leur responsabilité, les MJPM n'ont pas la disponibilité des familles.

Par ailleurs, c'est aux familles de malades mentaux de nous dire s'il était opportun d'ignorer la spécificité de ces personnes vulnérables en élargissant la catégorie des majeurs protégés. Attentif à la voix si singulière de l'UNAPEI ${ }^{112}$ parmi les quatre représentations nationales des associations tutélaires (UNAF, FNAT, CNAPE ${ }^{113}$ ), on serait porté à émettre un doute sérieux. Mais cette différence ne doit pas être exacerbée. Les MJPM ont, quel que soit le mode d'exercice de leurs missions et la singularité de leur objet associatif, plus de points communs ou de raisons de s'unir que de motifs de s'opposer.

Associés à la réflexion qui précède à l'élaboration d'une loi-programme sur les droits et l'éthique des personnes dépendantes et vieillissantes, les acteurs du système tutélaire et médico-social ainsi que les familles des personnes vulnérables ont le loisir de dénoncer les lacunes du dispositif juridique en vigueur et de proposer des remèdes appropriés. La consécration d'un statut juridique du professionnel «Mandataire judiciaire à la protection des majeurs» dans le Code de l'action sociale et des familles, d'une part, l'institution d'un Conseil national des MJPM chargé d'instruire, d'établir de manière contradictoire et de sanctionner les mauvais comportements professionnels, d'autre part, et l'élaboration d'un Code de déontologie des MJPM et des bonnes pratiques tutélaires, de troisième part, constitueraient trois nouvelles garanties offertes à la protection des majeurs vulnérables. La formulation de ce vœu est sous-tendue par un important travail de réflexion mené conjointement par des professionnels et des universitaires. Sa réalisation ne dépend maintenant plus que d'une volonté politique déterminée en ce sens.

112. Union nationale des parents d'enfants inadaptés. Outre les travaux de l'UNAPEI, on consultera avec intérêt les travaux du Comité des droits des personnes handicapées (CRPD en anglais: Committee on the Rights of Persons with Disabilities). Siégeant à Genève, cet organe est composé d'experts indépendants dont la mission est de surveiller l'application de la Convention internationale relative aux droits des personnes handicapées (CIDPH), adoptée à New York le 13 décembre 2006. Ce Comité est destinataire des rapports détaillés sur les mesures que les États membres ont prises pour se conformer aux obligations que leur impose la Convention, dans un délai de deux ans à compter de l'entrée en vigueur de la Convention (rapport initial) puis tous les quatre ans (rapport périodique). La France a signé la CIDPH le 30 mars 2007 et le Protocole facultatif le 23 septembre 2008, avant de ratifier la Convention et le Protocole facultatif le 18 février 2010. Ces deux textes sont entrés en vigueur en France le 20 mars 2010 .

113. Avec l'UNAPEI, l'Union nationale des associations familiales, la Fédération nationale des associations tutélaires et la Convention nationale des associations de protection de l'enfant sont les auteurs du Livre blanc sur la protection juridique des majeurs, septembre 2012. 